\title{
Bacteria-Targeted Fluorescence Imaging of Extracted Osteosynthesis Devices for Rapid Visualization of Fracture-Related Infections
}

\section{Marina López-Álvarez}

University of Groningen: Rijksuniversiteit Groningen

Marjolein Heuker

University of Groningen: Rijksuniversiteit Groningen

\section{Klaas A. Sjollema}

University Medical Centre Groningen: Universitair Medisch Centrum Groningen

Gooitzen M. van Dam

University of Groningen: Rijksuniversiteit Groningen

Jan Maarten van Dijl ( $\boldsymbol{Q}$ j.m.van.dijl01@umcg.nl )

Rijksuniversiteit Groningen https://orcid.org/0000-0002-5688-8438

\section{Frank F.A. IJpma}

University Medical Centre Groningen: Universitair Medisch Centrum Groningen

\section{Marleen van Oosten}

University Medical Centre Groningen: Universitair Medisch Centrum Groningen

\section{Research Article}

Keywords: fluorescence imaging, bone fracture, trauma, bacteria, infection, vancomycin

Posted Date: August 23rd, 2021

DOl: https://doi.org/10.21203/rs.3.rs-180623/v1

License: (c) (i) This work is licensed under a Creative Commons Attribution 4.0 International License.

Read Full License

Version of Record: A version of this preprint was published at European Journal of Nuclear Medicine and Molecular Imaging on January 26th, 2022. See the published version at https://doi.org/10.1007/s00259022-05695-y. 


\section{Abstract}

Purpose: Fracture-related infection (FRI) is a serious complication in orthopaedic trauma surgery worldwide. Especially the distinction of infection from sterile inflammation and the detection of low-grade infection are highly challenging. The objective of the present study was to explore the use of bacteriatargeted fluorescence imaging for enhanced detection of FRI on extracted osteosynthesis devices as a step-up towards real-time image-guided trauma surgery.

Methods: Extracted osteosynthesis devices from 13 patients, who needed revision surgery after fracture treatment, were incubated with a near-infrared fluorescent tracer composed of the antibiotic vancomycin and the fluorophore IRDye800CW (i.e. vanco-800CW). Subsequently, the devices were imaged and vanco$800 \mathrm{CW}$ fluorescence signals were correlated to the results of microbiological culturing and to bacterial growth upon replica plating of the imaged devices on blood agar.

Results: Importantly, compared to culturing, the bacteria-targeted fluorescence imaging of extracted osteosynthesis devices with vanco-800CW allows for a prompt diagnosis of FRI, reducing the time-toresult from days to less than $30 \mathrm{~min}$. Moreover, bacteria-targeted imaging will provide surgeons with realtime visual information on the presence and extent of infection.

Conclusion: Here we present the first clinical application of fluorescence imaging for detection of FRI. We conclude that imaging with vanco-800CW can provide early, accurate and real-time visual diagnostic information on FRI in the clinical setting, even in case of low-grade infections.

\section{Introduction}

Fracture-related infection (FRI) is a devastating complication after bone fracture treatment, resulting in severe patient morbidity and loss of quality of life. FRIs can be differentiated in 1) acute infections with clear signs of infection caused by highly pathogenic bacteria, and 2) low-grade infections caused by less pathogenic bacteria. Low-grade infections often lack specific clinical symptoms, which complicates their diagnosis. Treatment of FRI usually consists of revision surgery, including debridement and removal of infected bone and osteosynthesis devices (e.g. plates, nails and screws), combined with long-term antibiotic therapy $[1,2]$. During surgery, samples are collected for culture to verify infections, and identify causative micro-organisms for specific antibiotic therapy [2-4]. A major challenge is the distinction between non-infected and infected areas and devices during surgery, especially in case of low-grade infections. Surgeons rely on tactile and visual information to establish whether and to what extent wound areas are affected by the suspected infection. Further, a definitive pre-operative diagnosis of FRI is often not possible in case of low-grade infections due to the absence of unambiguous clinical symptoms of infection. In particular, the results from diagnostic modalities, such as white blood cell scintigraphy, fluorodeoxyglucose positron emission tomography (FDG-PET), or magnetic resonance imaging (MRI) are often inconclusive $[3,5,6]$. Therefore, a diagnostic imaging tool enabling early, accurate and real-time 
visualization of the infecting bacteria themselves, rather than inflammatory responses elicited by the infection, would provide a solution to this clinical problem.

Bacteria-targeted optical (i.e. fluorescence) imaging is an upcoming clinical imaging technique with great potential for the visualization of infections [7]. In particular, a conjugate of the antibiotic vancomycin and the near-infrared fluorophore IRDye800CW (i.e. vanco-800CW) was previously identified as an effective bacteria-targeted optical tracer in preclinical infection imaging studies [8, 9]. Like vancomycin, vanco$800 \mathrm{CW}$ specifically targets the Gram-positive bacterial cell wall. Since FRIs are mostly caused by Grampositive bacteria, such as Staphylococcus aureus, vanco-800CW seems particularly suited as a bacteriatargeted optical tracer for real-time diagnosis of FRI. Moreover, vanco-800CW efficiently binds Grampositive bacterial biofilms as encountered in FRI [10].

The overarching objective of our present study was to explore the possibility of using bacteria-targeted fluorescence imaging for enhanced detection of FRI on extracted osteosynthesis devices as a step-up towards real-time image-guided trauma surgery. The specific aim was to investigate whether vanco$800 \mathrm{CW}$ allows discrimination between infected and non-infected osteosynthesis devices, and whether it can be applied to visualize bacterial biofilms in clinical practice. To this end, we investigated osteosynthesis devices from 13 patients, who needed revision surgery and extraction of plates, screws and/or nails after unsuccessful prior fracture treatment. In addition, we addressed the question whether fluorescently labelled vancomycin allows the detection of vancomycin resistant Gram-positive bacteria.

\section{Materials And Methods}

\section{Fluorescence imaging of extracted osteosynthesis devices}

Osteosynthesis devices extracted from 13 trauma patients were included in this study. During surgery, the removed plates were divided in two by using nippers. One half of the plate was used for fluorescence imaging, the other half was used for regular diagnostics (described in the following section). A number of extracted screws, all retrieved from the same surgical site, were used for regular microbial diagnostics, and some for fluorescence imaging. Upon extraction, the osteosynthesis devices were washed with phosphate-buffered saline (PBS), and incubated with vancomycin-IRDye800CW (vanco-800CW, $0.14 \mathrm{nmol}$ $\mathrm{mL}^{-1}$, Li-COR Biosciences, Nebraska, USA) for $15 \mathrm{~min}$ at $37^{\circ} \mathrm{C}$. Subsequently, the incubated devices were washed twice with PBS to remove unbound vanco-800CW and imaged in the near-infrared range with an IVIS Lumina II imaging system (Excitation 710 nm, Emission filter ICG, Exposure times 1-10 s, PerkinElmer Inc., USA) and an intra-operative Explorer Air camera coupled to a closed-field imaging box (Vault, Excitation 760 nm, Emission filter Semrock FF01-819/44-25, Exposure times 100-200 ms, SurgVision B.V. Groningen, NL). To assess autofluorescence of the extracted device, it was imaged prior and post tracer incubation (Amersham Typhoon Biomolecular Imager, Filter IRLong 825BP30). To verify and visualize Gram-positive bacterial biofilms on extracted devices, microscopic imaging was performed with a twophoton confocal laser scanning microscope (objective 5x/0.16, filters 500-550/575-610, wavelength 850 $\mathrm{nm}$, Zeiss LSM 7MP) using vancomycin-BODIPY FL as a tracer $\left(0.14 \mathrm{nmol} \mathrm{mL}{ }^{-1}\right.$, Thermo Fisher Scientific, 
USA). Of note, the use of a two-photon microscope was necessary due to the shape and size of the materials tested (plates and screws) in order to have enough distance between the sample and objective. In turn, this required the application of vancomycin-BODIPY FL instead of vanco-800CW, because our twophoton microscope cannot image fluorescence in the near-infrared range. The fluorescence imaging workflow of extracted osteosynthesis devices is schematically represented in Figure 1.

\section{Sonication and culturing of extracted osteosynthesis devices}

The presence of microorganisms on the halved extracted plates and screws selected for regular diagnostics (see the afore-going section) and on collected tissues was independently investigated at the diagnostic microbiology laboratory of the University Medical Center Groningen (UMCG). Sonication of extracted osteosynthesis devices was performed as described by Trampuz et al. [11], with subsequent culturing of the sonicate on aerobic blood agar (BA), chocolate agar, anaerobic BA, and in blood culture bottles (BD BACTEC) as liquid culture. The decision whether osteosynthesis devices and/or tissues were infected was taken by the medical team, including a trauma surgeon, infectious disease specialist and a clinical microbiologist according to the standard protocol [4]. In addition, extracted osteosynthesis devices were replica plated on BA plates to correlate fluorescent signals to the presence of bacteria.

\section{Vancomycin-BODIPY FL binding to vancomycin resistant enterococci}

Vancomycin resistant Enterococcus faecium (VRE) strains carrying the vanA or vanB genes, vancomycin sensitive Enterococcus faecalis, and the Gram-negative control bacteria Escherichia coli and Klebsiella oxytoca were grown overnight in $3 \mathrm{~mL}$ Tryptic Soy Broth (TSB) at $37^{\circ} \mathrm{C}$ under constant agitation (250 RPM). Overnight cultures were diluted up to an optical density at $600 \mathrm{~nm}\left(\mathrm{OD}_{600}\right)$ of 2 in a final volume of $1 \mathrm{~mL}$. The bacteria were harvested by centrifugation for $3 \mathrm{~min}$ at 14.000 RPM. Subsequently, the bacteria were resuspended in $300 \mu \mathrm{L}$ of $0.14 \mathrm{nmol} \mathrm{mL}^{-1}$ vancomycin-BODIPY FL (Thermo Fisher Scientific, USA) and incubated for $15 \mathrm{~min}$ in the dark at $37^{\circ} \mathrm{C}$. The bacteria were then washed once with PBS to remove any unbound tracer and $100 \mu \mathrm{L}$ aliquots of each sample were transferred in triplicate to a flat-bottomed transparent 96-well plate for fluorescence measurements in a Biotek Synergy 2.0 plate reader (BioTek Instruments, Inc., USA). Fluorescence was measured with filters optimal for FITC, the excitation was set at $480 \mathrm{~nm}$ and emission at $520 \mathrm{~nm}$, with the optics position at 'bottom'. For control, triplicate samples of the bacteria incubated and washed only in PBS were also included in the plate. All measurements were carried out in triplicate at $37^{\circ} \mathrm{C}$ without shaking. In addition, fluorescence of the same 96-well plate was imaged with an Amersham Typhoon Biomolecular Imager (filter Cy2 525BP20).

\section{Data analysis and statistics}

Macroscopic fluorescence images and mean fluorescence intensity values were analyzed using ImageJ (National Institutes of Health, Maryland, USA) and Living Image 4.7.3 (PerkinElmer Inc, USA) software. Microscopic images were analyzed using Imaris 9.5.0 software. Regions of interest (ROIs) were drawn around the stained osteosynthesis devices after which the fluorescence signal was quantified. The background signal was quantified by drawing ROIs in uncontaminated biomaterials. Mann-Whitney U 
tests were used to determine the statistical significance of differences in fluorescence between infected or non-infected extracted devices and devices that had not been incubated with vanco-800CW. A p-value of $<0.05$ was considered statistically significant.

\section{Ethical approval}

Only surplus osteosynthesis devices that were considered unnecessary for clinical diagnosis were used for the present bacteria-targeted imaging with vanco-800CW or vancomycin-BODIPY FL in accordance with the permission obtained from the Medical Ethical Review Board of the UMCG (permission number METc 2016/481). All experiments were performed with adherence to the guidelines of the Declaration of Helsinki and local regulations. Patient data were used pseudo-anonymously based on informed consent.

\section{Results}

The investigated patient cohort consisted of 13 patients, who underwent revision surgery in the UMCG between January 2018 and February 2020 to extract osteosynthesis devices after bone fracture treatment. Patients were either operated upon clinical suspicion of FRI (i.e. redness, swelling, persistent wound-leakage, non-union, plate breakage, $n=9$, Figures 2 and 3) or for removal of osteosynthesis devices for mechanical reasons ( $n=4$, culture-negative controls, Figure 4). Altogether, 59 extracted osteosynthesis devices (i.e. screws, plates and nails) were treated with vanco-800CW and imaged macroscopically as schematically represented in Figure 1 . To verify and visualize the presence of Grampositive bacterial biofilms, particular extracted devices were also imaged in triplicate by fluorescence microscopy using vancomycin-BODIPY FL as a tracer. Importantly, evaluation of the imaging results obtained with the extracted osteosynthesis devices from all patients included in the present proof-ofprinciple study with vanco-800CW allowed a clear distinction of infected (Figures 2 and 3 ) and noninfected devices (Figure 4), as was independently verified by microbiological culturing. Of note, Figure 2 also presents the raw fluorescence images (labelled as 'surgeon's view') that can be intra-operatively viewed by the surgeon. Statistical analysis of the imaging results obtained for all 59 extracted osteosynthesis devices treated with vanco-800CW showed significant differences between infected and non-infected devices (Figure 5). Importantly, the vanco-800CW fluorescence signal could be easily distinguished from light reflections and the autofluorescence of the extracted devices themselves, as illustrated in Figure 3. In the following sections, the visualization of FRI by staining extracted osteosynthesis devices with vanco- $800 \mathrm{CW}$ is documented in detail through two clinical cases of, respectively, acute and low-grade infections.

\section{Bacteria-targeted imaging in an infected non-union of a tibial fracture}

A first clinical example of successful bacteria-targeted fluorescence imaging with vanco-800 CW in a clinical setting was obtained for a patient with tibial non-union accompanied with acute FRI (Figure 6). In this case, a 51-year-old man (patient 11) suffered a lower leg fracture for which he was initially treated with a cast. After 7 months, barely any fracture healing of the tibia had occurred and therefore operative debridement of the non-union site, followed by a cancellous bone graft and tibial plate fixation was 
performed. The post-operative course was complicated by FRI, characterized by wound dehiscence and eventually an exposed implant (Figure 6A, B). Therefore, the infected plate and screws had to be removed. The plate was surrounded by debris and minimal cloudy seroma (Figure 6C).

Figures $6 \mathrm{C}$ and $6 \mathrm{D}$ respectively show the tibial plate in situ, upon macroscopic analysis after extraction and $15 \mathrm{~min}$ incubation with vanco-800 CW. The latter revealed a clear fluorescence signal with a patchy distribution (Figures 6E-G, marked 'POS'), while control osteosynthesis devices of the confirmed infectionnegative patient 10 emitted no fluorescence signals (Figures 6E-G, marked 'NEG'). To verify and visualize the presence of Gram-positive bacterial biofilms, extracted screws were stained with vancomycin-BODIPY FL and imaged with a two-photon microscope. Indeed, biofilm formation was clearly visualized, demonstrating the high binding specificity of vancomycin-based tracers (Figure $6 \mathrm{H}$, I, for Video, see supplementary data, Movie S1). Sonication of extracted biomaterials and subsequent microbiological culturing identified the Gram-positive bacteria Corynebacterium tuberculostearicum, Staphylococcus aureus and Staphylococcus epidermidis as causative agents of the infection.

\section{Detection of low-grade FRI with vanco-800CW}

The high sensitivity and specificity of bacteria-targeted optical imaging with vanco-800CW is supported by a second case, where a patient with a fracture-related low-grade infection of the femur presented no clinical symptoms of infection prior surgery (patient 5 , Figure 7A). The X-ray showed a non-union of a femoral fracture with a broken plate (Figure 7B). During surgery, no signs of bacterial infection were observed (Figure 7C, D, F). Nonetheless, relatively low but clear fluorescence signals were detectable upon 15 min incubation of the extracted plate and screws with vanco-800CW (Figure 7E, G, I). Moreover, the fluorescence signals were directly correlated to bacterial growth upon replica plating of the extracted screws on BA plates (Figure 7H-J). Microbiological culturing revealed that osteosynthesis devices of this patient carried the Gram-positive bacterium Cutibacterium (Propionibacterium) acnes.

\section{Detection of vancomycin-resistant Gram-positive bacteria}

Lastly, to investigate whether the use of vancomycin-based tracers could be limited by increased vancomycin resistance in Gram-positive bacteria, we assessed the binding of vancomycin-BODIPY FL to clinical vanA-or vanB-positive $E$. faecium isolates that are resistant to vancomycin (VRE), and a vancomycin sensitive $E$. faecalis control isolate. In addition, we used the Gram-negative bacteria $E$. coli and K. oxytoca as controls. As shown in Figure 8, the VRE isolates bound significantly more vancomycin-BODIPY FL than the Gram-negative control strains, although the binding of this tracer by the VRE isolates was reduced compared to the vancomycin sensitive $E$. faecalis isolate. Altogether, these observations imply that vancomycin-based optical tracers do allow the detection of vancomycin resistant Gram-positive bacteria.

\section{Discussion}


The present clinical investigation shows that detection of bacterial growth on osteosynthesis devices by optical imaging with vanco-800CW is highly specific, sensitive, fast and feasible in the clinical setting. On the contrary, the current diagnosis of FRI relies heavily on culture-based methods that may take several days. Hence, patients with suspected FRI are often treated empirically with revision surgery and broadspectrum antibiotics until the causative organism(s) have been identified. Here we show that imaging of potentially infected osteosynthesis devices with vanco-800 CW reduces the time-to-result from days to less than $30 \mathrm{~min}$. This implies that bacteria-targeted fluorescence imaging may significantly enhance intra-operative clinical decision-making regarding empirical antibiotic treatment, extensive debridement, the choice between one- and two-stage revision surgery, or implant exchange [12]. A possible limitation of the fluorescence imaging approach with vanco-800 CW is that it cannot replace current diagnostic procedures, because it does not identify the causative agents of FRI and the associated antibiotic resistances. Hence, we regard optical imaging with vanco-800CW as a complementary tool to the current diagnostic methods for prompt visualization of an infection. Ideally, a definitive diagnosis of the causative agent is needed to direct antibiotic treatment, which may be achieved in the future by the use of multiple tracers next to vanco- $800 \mathrm{CW}$, i.e. targeting Gram-negative bacteria, or specific bacterial species.

Based on the present results, we envisage that intra-operative topical application of vanco-800CW, incubation and rinsing off excess and unbound tracer during surgery may provide surgeons with real-time visual information on the presence and extent of infection. Here it is noteworthy that imaging can be performed with doses of vanco-800 CW that are 20-40-fold below the minimal inhibitory concentration (MIC) of vancomycin for staphylococci (https://eucast.org/clinical_breakpoints/) [8, 10]. Thus, the results of routine diagnostic procedures and culturing will not be affected by the imaging procedure. Another clear advantage of vanco- $800 \mathrm{CW}$ is its fluorescence in the near-infrared range, which minimizes interference by tissue autofluorescence $[6,10,13,14]$. In our study, an IVIS imaging system and an intraoperative camera coupled to a closed-field imaging box were used to detect the emitted fluorescence signal. The latter camera system is already in use for surgical applications in the operating theater $[15$, 16]. Yet, prior to any intra-operative application of vanco-800CW, it will be necessary to assess its safety, although vancomycin [17] and the IRDye800CW fluorophore have separately been approved for clinical implementation $[18,19]$. A second limitation of vanco-800CW is that it will probably not detect FRI caused by Gram-negative bacteria, as exemplified in the present study by the lack of vancomycin-BODIPY FL binding to $E$. coli and $K$. oxytoca. This is in accordance with the fact that vancomycin shows the highest specificity for Gram-positive bacterial cell walls. However, Gram-negative bacteria cause $\sim 30 \%$ of all cases [20], which implies that vanco-800CW will allow a correct diagnosis of FRI in most cases. This view is supported by the present results and, in particular, by the observation that even notoriously vancomycin resistant bacteria, such as VRE, can bind vancomycin-based tracers.

Both from the diagnostic and scientific perspectives, it would be interesting if the fluorescent signals detected with tracers like vanco-800CW could be used to quantify the numbers of viable bacteria on extracted osteosynthesis devices, and to determine the cut-off lines for positive testing of infection. However, a reliable bacterial quantification on extracted implants is technically challenging, if not impossible. In the first place, different Gram-positive bacterial species that cause implant-associated 
infections will bind vancomycin with different efficiencies, and this also applies to vanco-800CW as we have previously demonstrated [9]. Hence, as long as the causative infecting organism has not been identified, it is not possible to correlate vanco-800CW-specific fluorescence to numbers of bacterial colony-forming units (CFUs). This becomes even more challenging in the case of polymicrobial infections that are frequently encountered in clinical settings as underscored by the cases portrayed in Figures 2 and 3. Secondly, vanco-800 CW will also bind to the cell walls of dead bacteria, which adds to the challenge of correlating fluorescence to CFUs in a clinical setting [9]. A third hurdle relates to the fact that bacterial biofilms are not homogeneously distributed over an explanted osteosynthesis device, whereas part of the explanted device needs to be used for routine clinical diagnostics analyses to identify the causative microorganisms and their antibiotic resistances. The latter is of prime importance with respect to patient care and associated ethical considerations. Accordingly, for the present experimental study, upon surgery extracted osteosynthesis devices had to be divided and separately investigated with priority for clinical diagnostic testing, including sonication and subsequent microbiological culturing. However, this approach is semi-quantitative and the results can be used to validate, but not to quantify the detected bacteria on the samples used for imaging with vanco-800CW. Nonetheless, despite the fact that the precise cut-off lines for test positivity based on fluorescence are not yet known, our present study shows that the main strength of detecting osteosynthesis device infections with vanco-800CW lies in: 1) its high discriminatory power between infected and non-infected devices, 2) the short time it takes to visualize bacteria on extracted devices, and 3 ) the possibility to take rapid informed decisions concerning the further treatment of patients during surgery. Moreover, the collected images provide unique insights in the distribution of bacterial biofilms on infected osteosynthesis materials in patients.

Lastly, our present study demonstrates the first successful clinical application of bacteria-targeted fluorescence imaging for the rapid visualization of fracture-related infections. We therefore conclude that bacteria-targeted optical imaging with vanco-800CW provides new perspectives in the fight against FRI. This technique allows early, accurate and real-time diagnosis of FRI in the clinical setting, even in case of low-grade infections. Moreover, our findings suggest that bacteria-targeted imaging may serve as a highly sensitive tool for rapid detection of implant infections, ranging from osteosynthesis devices to surgical meshes, stents, prosthetic valves and vascular grafts. While the present study was exclusively based on the use of fluorescent tracers for the optical imaging of infection, we anticipate that future applications will involve also radioactively labelled bacteria-targeted tracers for the non-invasive diagnosis of FRI and other deep-seated implant infections [5-7, 21].

\section{Declarations}

Funding: M.L-A was funded by Marie Skłodowska-Curie Actions grant number 713660 PRONKJEWAIL. M.H. was funded by the Graduate School of Medical Sciences of the University of Groningen.

Conflict of interest: GMvD is consultant for OncoNano Medicine Inc. and CEO, founder and shareholder of the AxelaRx / TRACER BV group. The other authors have no conflicts of interest to disclose. 
Availability of data and material: All data are provided with the manuscript, except data that identify the included patients. Vanco-800CW was purchased from Li-COR Biosciences, Nebraska, USA.

Code availability: Not applicable.

Author contributions: ML-A, MH, GMvD, JMvD, FFAIJ, and MvO conceived the study. JMvD, FFAIJ and MvO supplied materials. ML-A and MH performed the experiments. ML-A and KAS performed the microscopy. ML-A, MH, JMvD, FFAIJ and MvO wrote the manuscript. All authors reviewed and approved the manuscript.

Ethical approval: Permission for this study was granted by the local Medical Ethical Review Board (Medisch Ethische Toetsingscommissie, METc, number 2016/481) for the use of surplus osteosynthesis devices and clinical data, that would not interfere with the diagnostic process. The research was performed with adherence to the guidelines of the Declaration of Helsinki.

Consent to participate: Patients gave informed consent, which included the use of pseudo-anonymized data and samples for the purpose of research and publication.

Consent for publication: All authors have read the manuscript and agree with its publication.

\section{Acknowledgments}

We thank Pieter Jan Steinkamp for help with the image analysis, the department of Biomedical Engineering at the University Medical Center Groningen, in particular Jelmer Sjollema, for access to the IVIS Lumina II, Ed de Jong for excellent technical assistance, and Xuewei Zhou for providing vancomycin resistant Enterococcus faecium isolates.

\section{References}

1. Engelsman AF, Saldarriaga-Fernandez IC, Nejadnik MR, van Dam GM, Francis KP, Ploeg RJ, et al. The risk of biomaterial-associated infection after revision surgery due to an experimental primary implant infection. Biofouling. 2010,26(7):761-7.

2. Morgenstern M, Kühl R, Eckardt H, Acklin Y, Stanic B, Garcia M, et al. Diagnostic challenges and future perspectives in fracture-related infection. Injury. 2018,49 Suppl 1:S83-S90.

3. Govaert GAM, Kuehl R, Atkins BL, Trampuz A, Morgenstern M, Obremskey WT, et al. Diagnosing Fracture-Related Infection: Current Concepts and Recommendations. J. Orthop Trauma. 2020,34(1):8-17.

4. Metsemakers WJ, Morgenstern M, McNally MA, Moriarty TF, McFadyen I, Scarborough M, et al. Fracture-related infection: A consensus on definition from an international expert group. Injury. 2018,49(3):505-510.

5. Ordonez AA, Sellmyer MA, Gowrishankar G, Ruiz-Bedoya CA, Tucker EW, Palestro CJ et al. Molecular imaging of bacterial infections: Overcoming the barriers to clinical translation. Sci Transl Med. 
2019,4,11(508):eaax8251.

6. van Oosten M, Hahn M, Crane LMA, Pleijhuis RG, Francis KP, van Dijl JM, et al. Targeted imaging of bacterial infections: Advances, hurdles and hopes. FEMS Microbiol. 2015,39(6):892-916.

7. Heuker M, Gomes A, van Dijl JM, van Dam GM, Friedrich AW, Sinha B, et al. Preclinical studies and prospective clinical applications for bacteria-targeted imaging: the future is bright. Clin Transl Imaging. 2016,4:253-264.

8. Reeßing F, Bispo M, López-Álvarez M, van Oosten M, Feringa BL, van Dijl JM et al. A Facile and reproducible synthesis of near-infrared fluorescent conjugates with small targeting molecules for microbial infection imaging. ACS Omega. 2020,26,5(35):22071-22080.

9. van Oosten M, Schäfer T, Gazendam JAC, Ohlsen K, Tsompanidou E, De Goffau MC, et al. Real-time in vivo imaging of invasive- and biomaterial-associated bacterial infections using fluorescently labelled vancomycin. Nat Commun. 2013,4:2584.

10. Schoenmakers JWA, Heuker M, López-Álvarez M, Nagengast WB, van Dam GM, van Dijl JM, et al. Image-guided in situ detection of bacterial biofilms in a human prosthetic knee infection model: a feasibility study for clinical diagnosis of prosthetic joint infections. Eur. J. Nucl. Med. Mol. Imaging. 2020,8 .

11. Trampuz A, Zimmerli W. Diagnosis and treatment of infections associated with fracture-fixation devices. Injury. 2006,37 Suppl 2:S59-66.

12. Voskuil FJ, Steinkamp PJ, Zhao T, van der Vegt B, Koller M, Doff JJ, et al. Exploiting metabolic acidosis in solid cancers using a tumor-agnostic $\mathrm{pH}$-activatable nanoprobe for fluorescence-guided surgery. Nat Commun. 2020,26,11(1):3257.

13. Joshi BP, Wang TD. Targeted Optical Imaging Agents in Cancer: Focus on Clinical Applications. Contrast Media Mol Imaging. 2018,27,2018:2015237.

14. Mills B, Bradley M, Dhaliwal K. Optical imaging of bacterial infections. Clin Transl Imaging. 2016,4:163-174.

15. Steinkamp PJ, Pranger BK, Li M, Linssen M, Voskuil FJ, Been L, et al. Fluorescence-guided visualization of soft tissue sarcomas by targeting vascular endothelial growth factor-A: a phase 1 single-center clinical trial. J Nucl Med. 2020,jnumed.120.245696.

16. van Dam GM, Themelis G, Crane LMA, Harlaar NJ, Pleijhuis RG, Kelder W, et al. Intraoperative tumorspecific fluorescence imaging in ovarian cancer by folate receptor-a targeting: First in-human results. Nat Med. 2011,18,17(10):1315-9.

17. Rubinstein E, Keynan Y. Vancomycin revisited - 60 years later. Front Public Health. 2014,31,2:217.

18. de Jongh SJ, Tjalma JJJ, Koller M, Linssen MD, Vonk J, Dobosz M, et al. Back-Table FluorescenceGuided Imaging for Circumferential Resection Margin Evaluation Using Bevacizumab-800CW in Patients with Locally Advanced Rectal Cancer. J. Nucl. Med. 2020,61(5):655-661.

19. Voskuil FJ, de Jongh SJ, Hooghiemstra WTR, Linssen MD, Steinkamp PJ, de Visscher SAHJ, et al. Fluorescence-guided imaging for resection margin evaluation in head and neck cancer patients 
using cetuximab-800CW: A quantitative dose-escalation study. Theranostics. 2020,4,10(9):39944005.

20. Maleb A, Frikh M, Lahlou YB, Chagar B, Lemnouer A, Elouennass M. Bacteriological aspects of chronic osteoarticular infections in adults: The influence of the osteosynthesis material. BMC Res Notes. 2017,28,10(1):635.

21. Pinkston KL, Singh KV, Gao P, Wilganowski N, Robinson H, Ghosh S, Azhdarinia A, Sevick-Muraca EM, Murray BE, Harvey BR. Targeting pili in enterococcal pathogenesis Infect Immun. 2014,82(4):15401547.

22. Zoller SD, Park HY, Olafsen T, Zamilpa C, Burke ZD, Blumstein G, Sheppard WL, Hamad CD, Hori KR, Tseng JC, Czupryna J, McMannus C, Lee JT, Bispo M, Romero Pastrana F, Raineri EJ, Miller JF, Miller LS, van Dijl JM, Francis KP, Bernthal NM. Multimodal imaging guides surgical management in a preclinical spinal implant infection model. JCI Insight. 2019,4(3):e124813.

\section{Figures}




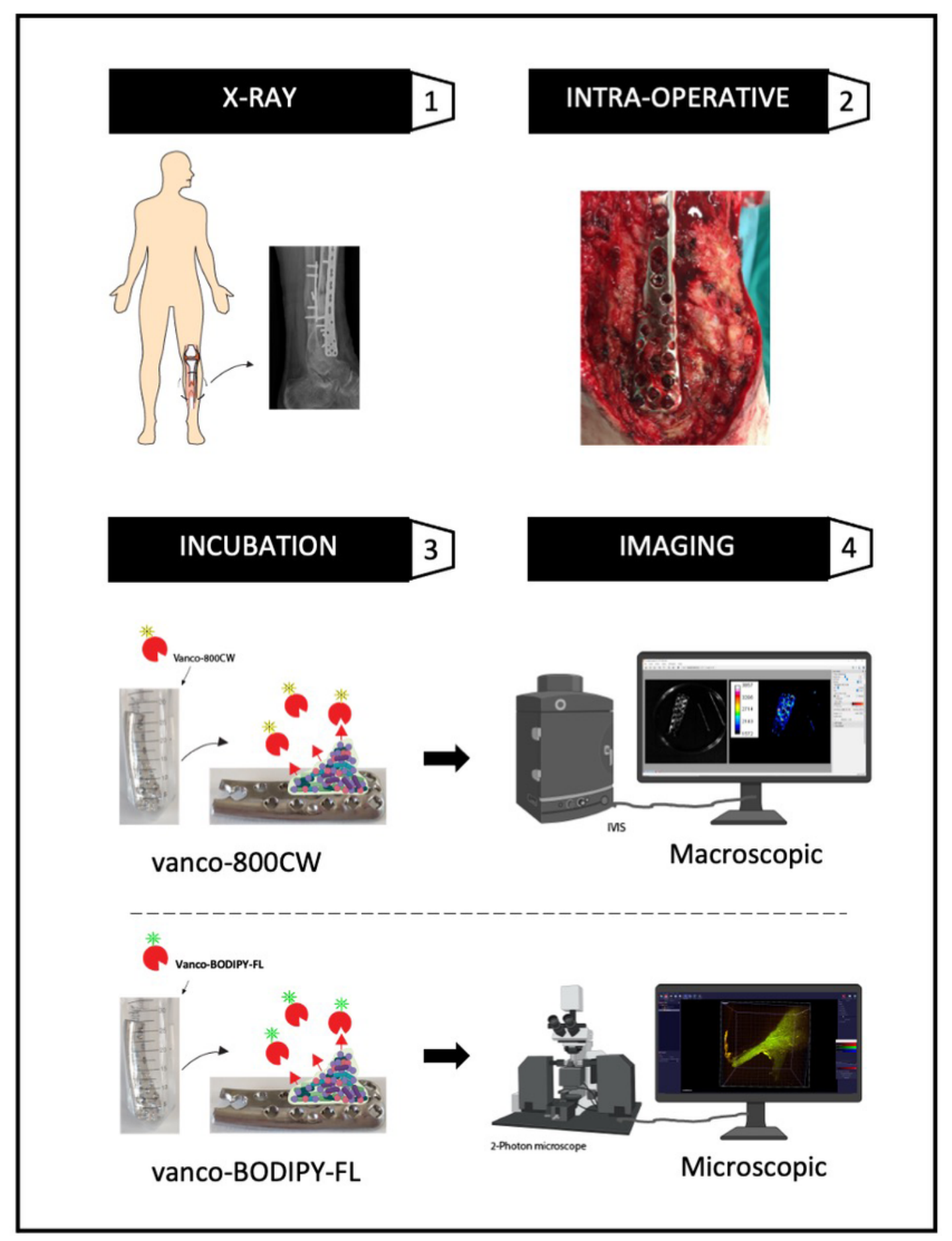

Figure 1

Schematic overview of the fluorescence imaging workflow. Osteosynthesis devices extracted during surgery were washed with PBS and incubated with vanco-800CW $(0.14 \mathrm{nmol} / \mathrm{mL})$ for $15 \mathrm{~min}$ at $37 \mathrm{oC}$. Subsequently, the incubated devices were washed twice with PBS to remove unbound vanco-800CW and imaged macroscopically with an IVIS Lumina II imaging system and an intra-operative Explorer Air camera coupled to a closed-field imaging box (Vault). To verify and visualize the presence of bacterial 
biofilms, for some osteosynthesis devices additional microscopic imaging was performed with a twophoton confocal laser scanning microscope using vancomycin-BODIPYTM FL as a tracer. Part of this figure was created with BioRender.com.

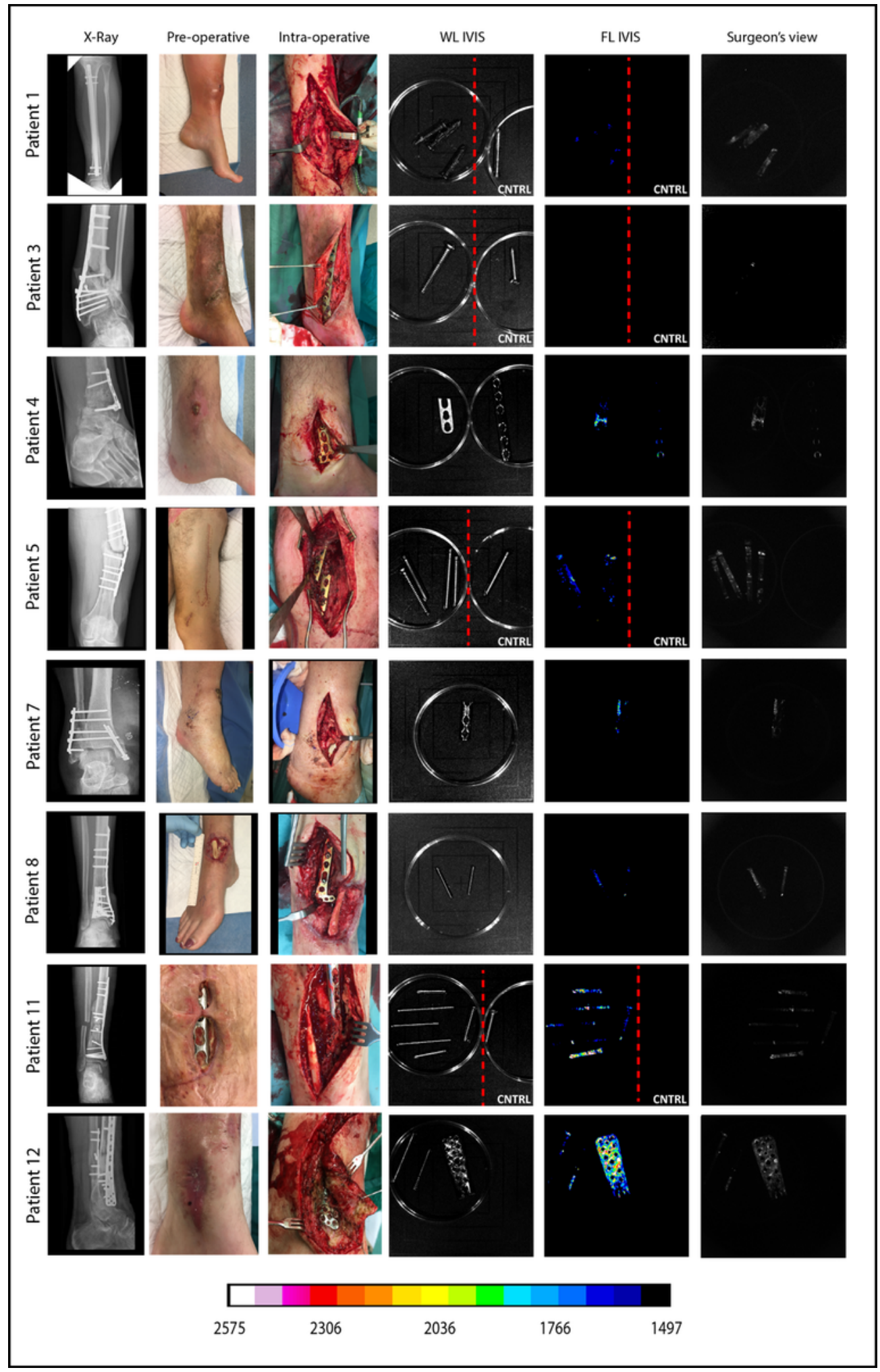

Figure 2

Bacteria-targeted fluorescence imaging of extracted osteosynthesis devices from patients with microbiologically confirmed fracture-related infections. Corresponding X-ray, pre-operative, intra-operative, 
white light (WL IVIS) and fluorescence (FL IVIS) images (IVIS Lumina II imaging system, PerkinElmer Inc., USA, Excitation $710 \mathrm{~nm}$, Emission filter ICG, Exposure time $10 \mathrm{sec}$ ) of 8 patients and their extracted osteosynthesis devices with a microbiologically confirmed fracture-related infection (FRI) after surgery. The right column shows the raw images as can be viewed by the surgeon (labelled as 'Surgeon's view'). The presence of microorganisms on extracted osteosynthesis devices was investigated by culturing in the diagnostic microbiology laboratory of the University Medical Center Groningen, independently from the bacteria-targeted imaging with vanco-800CW. Patient 1. Diagnosis: FRI after intramedullary nailing of a crural fracture. Clinical signs: local redness and swelling with elevated inflammation markers (Creactive protein $347 \mathrm{mg} / \mathrm{L}$ ). Surgery: removal of the nail, local debridement of the pretibial abscess and cavitary defect of the tibia. Culture: low growth density of Streptococcus anginosus (Gram-positive). Imaging: positive, however, weak signal due to a low bacterial density. Patient 3. Diagnosis: infected nonunion of the distal tibia with plate breakage. Clinical signs: scar tissue on the medial side of the lower leg with some redness. Surgery: removal of the broken plate and screws, debridement and washout of the non-union site, temporary antibiotic cement spacer (Masquelet procedure) and application of an external fixator. Culture: Enterobacter cloacae (Gram-negative) and Staphylococcus epidermidis (Gram-positive) both in very low densities. Imaging: positive, however, weak signal due to low Gram-positive bacterial density. Patient 4. Diagnosis: FRI after multiple operations of a comminuted distal tibial fracture. Clinical signs: redness and fistula at the medial malleolus. Surgery: excision of the fistula, removal of osteosynthesis devices, local debridement and washout. Culture: Acinetobacter radioresistens (Gramnegative), Citrobacter koseri (Gram-negative), Corynebacterium aurimucosum (Gram-positive), Corynebacterium jeikeium (Gram-positive), Dermabacter hominis (Gram-positive), Staphylococcus haemolyticus (Gram-positive). Imaging: positive. Patient 5. Diagnosis: infected non-union of the left femur with a plate breakage. Clinical signs: no clinical signs of infection at the lateral thigh. Surgery: removal of the osteosynthesis devices, debridement of the non-union site and reosteosynthesis with an intramedullary nail. Culture: Cutibacterium (Propionibacterium) acnes (Gram-positive). Imaging: positive. Note that these data are also included in Figure 7. Patient 7. Diagnosis: FRI after osteosynthesis of a Gustillo grade 3 complicated ankle fracture. Clinical signs: pain, swelling, redness, septic arthritis, elevated inflammation markers (CRP $280 \mathrm{mg} / \mathrm{L}$ ). Surgery: removal of the osteosynthesis devices, washout of the ankle joint, reosteosynthesis of the medial malleolus. Culture: Staphylococcus aureus (Gram-positive). Imaging: positive. Patient 8. Diagnosis: FRI after osteosynthesis of a distal tibial fracture. Clinical signs: wound dehiscence with partially exposed plate. Surgery: removal of ventral plate and washout of the wound. Culture: Enterococcus faecalis (Gram-positive) and Staphylococcus aureus (Gram-positive). Imaging: positive. Patient 11. Diagnosis: infected non-union of a crural fracture. Clinical signs: wound dehiscence and exposed implant. Surgery: removal of infected osteosynthesis devices and a washout of the non-union site. Culture: Staphylococcus aureus (Gram-positive). Imaging: positive. Note that these data are also included in Figure 6. Patient 12. Diagnosis: infected non-union of the distal tibia. Clinical signs: local redness, swelling around the scar on the medial malleolus. Surgery: removal of plates and screws, debridement of the non-union, temporary antibiotic cement spacer (Masquelet procedure), soft tissue coverage with a latissimus dorsi flap. Culture: Enterococcus faecalis (Gram-positive), 
Staphylococcus capitis (Gram-positive) and Staphylococcus epidermidis (Gram-positive). Imaging: positive. Images were analyzed with ImageJ and Living Image 4.7.3 software.
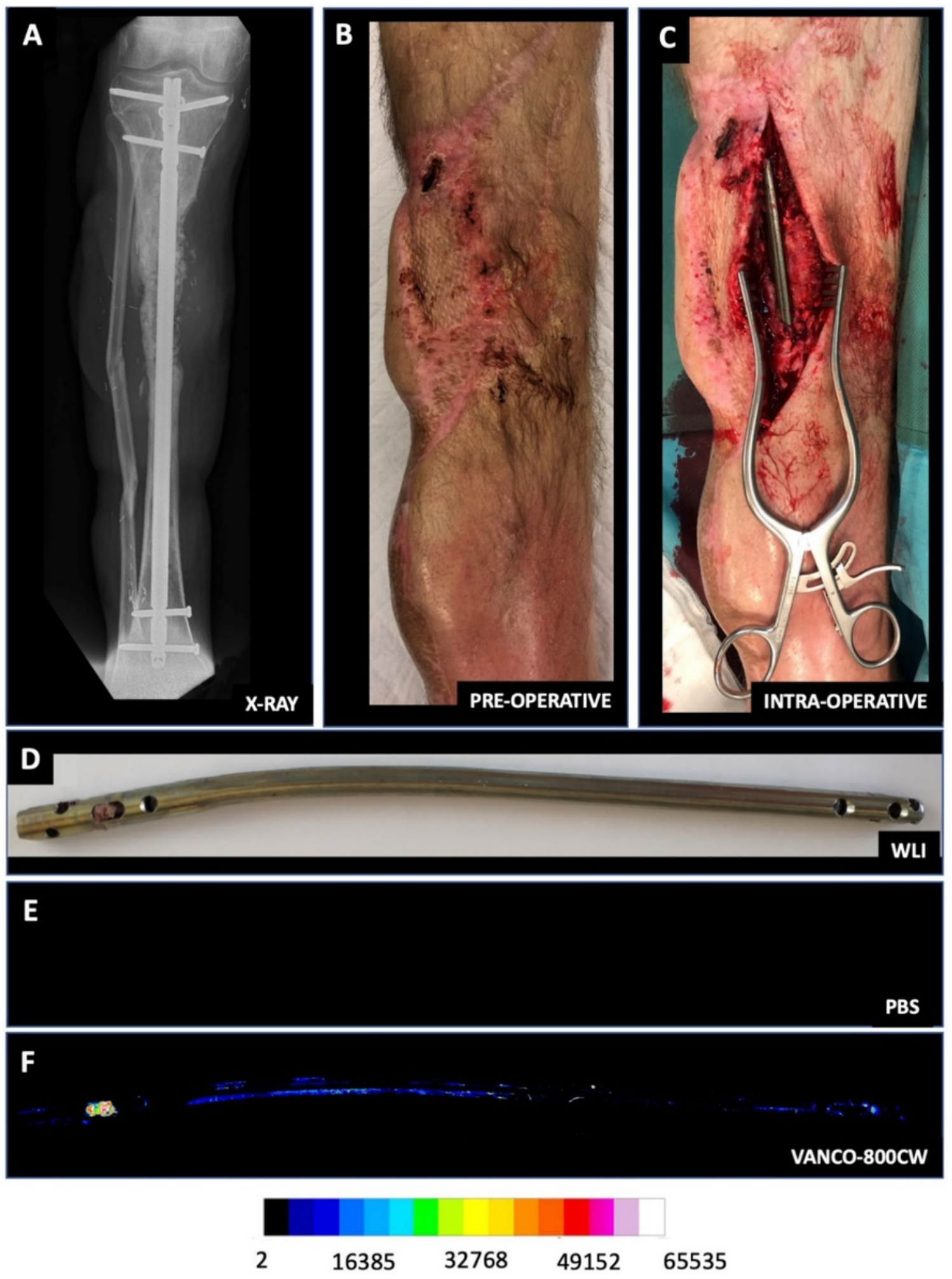

Figure 3

Fluorescence imaging of an extracted tibia nail from a patient with microbiologically confirmed fracturerelated infection prior and post incubation with vanco-800CW. Patient 13, Diagnosis: infected non-union of the proximal tibia after multiple operations for a complicated crural fracture. Clinical signs: redness, 
swelling and fistula proximal tibia. Surgery: removal of the nail, debridement and washout of the nonunion site, temporary antibiotic cement spacer (Masquelet procedure). Culture: Staphylococcus aureus (Gram-positive) and Cutibacterium acnes (Gram-positive). Imaging: positive. The upper panels show an $X$-ray image of the patient's lower leg (A), the pre-operative clinical presentation (B), and an intra-operative image with the infected tibia nail prior extraction (C). D. White light (WL) image of the extracted tibia nail. E. Fluorescence image prior incubation with vanco-800CW. F. Fluorescence image of the tibia nail after 15 min of incubation with vanco-800CW and subsequent washing with PBS. Images were taken with an Amersham Typhoon Biomolecular Imager (Filter IRLong 825BP30) and analyzed with ImageJ software. 


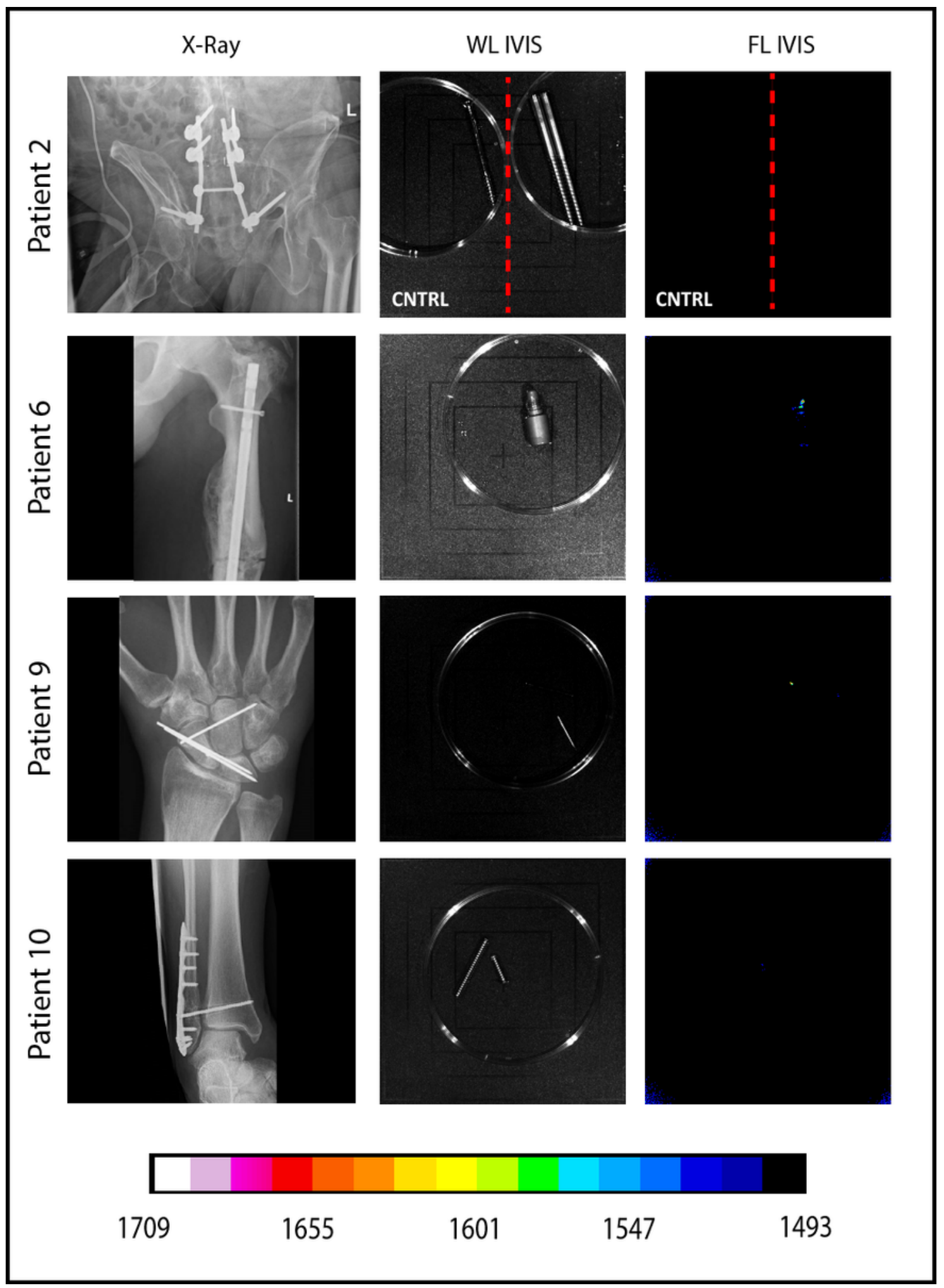

\section{Figure 4}

Bacteria-targeted fluorescence imaging of four confirmed culture-negative osteosynthesis devices. Corresponding X-ray, white light (WL IVIS) and fluorescence (FL IVIS) images (IVIS Lumina II imaging system, PerkinElmer Inc., USA, Excitation 710 nm, Emission filter ICG, Exposure time $10 \mathrm{sec}$ ) of four patients without detectable implant infections. The absence of microorganisms on extracted osteosynthesis devices was concluded based on culturing in the diagnostic microbiology laboratory of 
our hospital, independently from the bacteria-targeted imaging with vanco-800CW. None of the patients showed clinical symptoms of inflammation and all imaging results of extracted osteosynthesis devices incubated with vanco-800CW were negative. Patient 2. Extracted lumbo-pelvic fixation device due to lower back pain. Patient 6 . Removed endcap of a femur nail during exchange nailing for a non-union of a femoral fracture. Patient 9. Routinely removed K-wires of the right wrist after treatment of carpal injuries. Patient 10. Routinely removed lag screw after open reduction internal fixation of a Weber $C$ ankle fracture. Images were analyzed with ImageJ and Living Image 4.7.3 software.

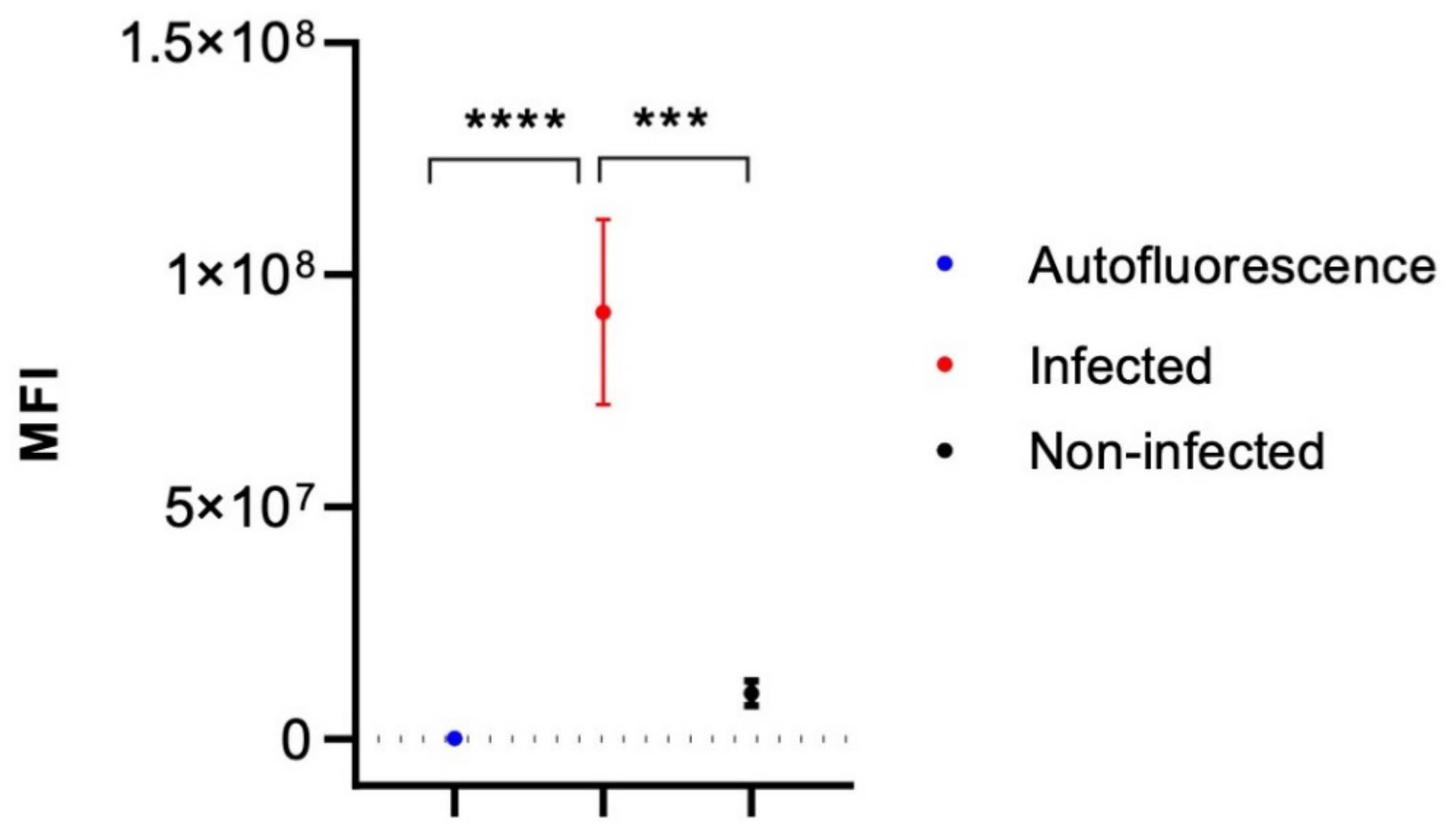

Figure 5

Mean Fluorescence Intensity (MFI) values of all extracted osteosynthesis materials imaged with vanco$800 \mathrm{CW}$. Comparison of the MFI values from all 59 extracted osteosynthesis devices imaged with vanco$800 \mathrm{CW}$ shows a significantly increased MFI of materials from patients with an infection (red) compared to materials from patients without infection (black). Moreover, a significant difference was observed between the mean autofluorescence intensity measured in the absence of tracer (blue) and the MFI of infected materials incubated with vanco-800 CW. Mann-Whitney $U$ tests were used for statistical analyses. Significant differences between groups are marked ( $\star \star \star \star, P<0.0001, \star \star \star, P=0.0001)$. Values represent the mean \pm SEM. 

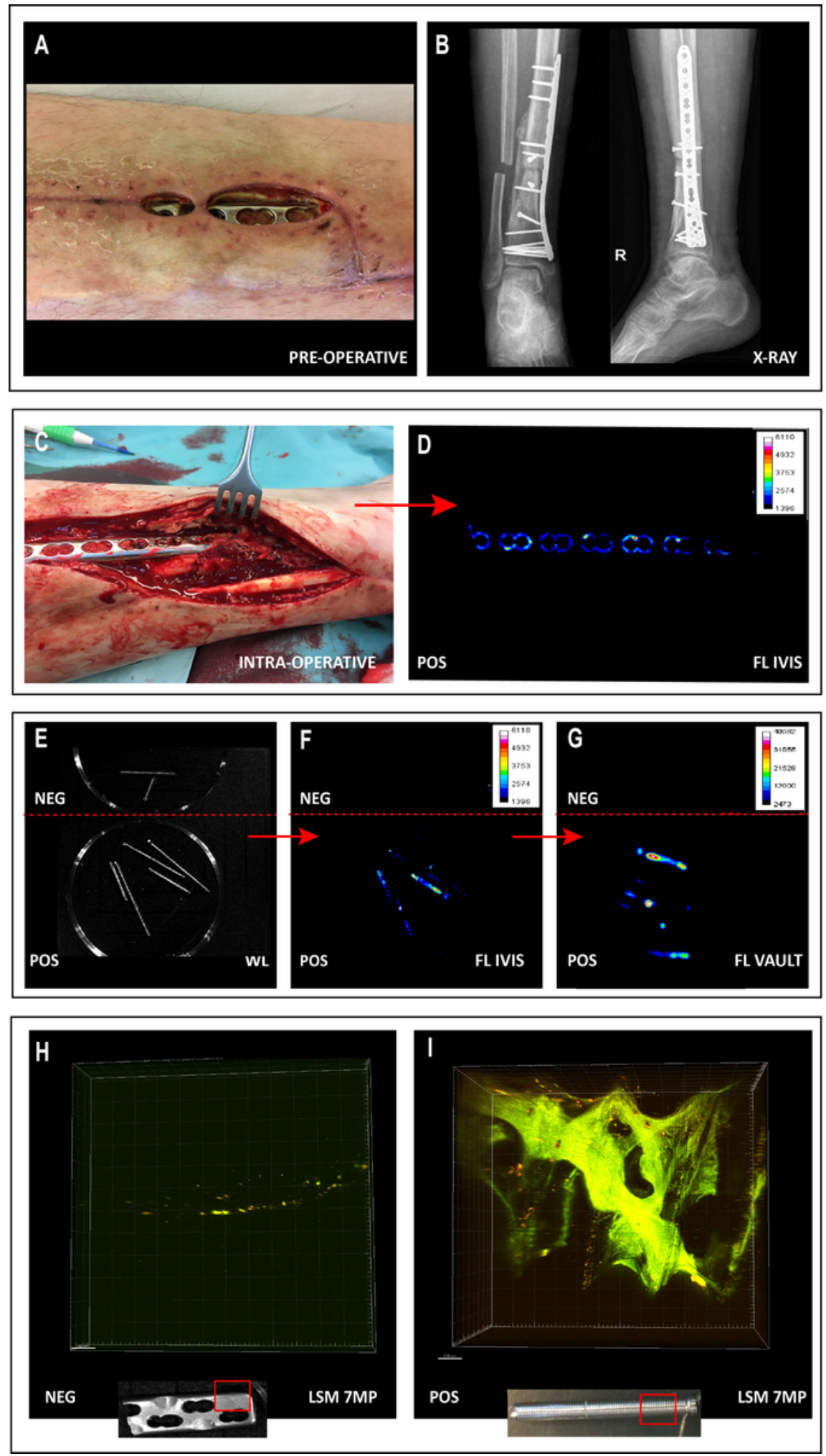

\section{Figure 6}

Bacteria-targeted imaging with fluorescently labeled vancomycin of a fracture-related lower leg implant infection. Images relate to patient 11 unless indicated otherwise. A. Pre-operative clinical presentation with wound dehiscence and an exposed plate of the lower leg. B. X-ray of the delayed union of a crural fracture with tibial plate fixation. C. Intra-operative image showing the infected tibial plate prior extraction. D. Macroscopic fluorescence image (FL IVIS) of the extracted plate upon staining with vanco-800CW. E. 
White light (WL) image of both infected (POS) and confirmed culture-negative (NEG) extracted screws. Note that the culture-negative screws were obtained from a different patient (patient 10, Figure 4), but imaged simultaneously with infected screws of patient 11. F,G. Fluorescence images of the screws presented in E upon staining with vanco-800CW performed with two different imaging devices, respectively an IVIS Lumina II (F. PerkinElmer Inc., USA, Excitation 710 nm, Emission filter ICG, Exposure time $10 \mathrm{~s}$ ) and an intra operative camera (G. Explorer Air coupled to a closed-field imaging box [Vault], SurgVision B.V. Groningen, NL, Excitation 760 nm, Emission filter Semrock FF01-819/44-25 nm, Exposure times 100-200 ms). Of note, there is a slight mismatch in the fluorescence signals in the images of panels $F$ and $G$, which relates to slightly different positions of the imaged screws in the IVIS and the intra operative camera system. Further, different settings were applied in the two systems (i.e. a binning of 4 in the IVIS and a binning of 1 in the Vault), which explains the differences in pixel size. H,I. Microscopic image of a negative control $(\mathrm{H})$ and infected screw $(\mathrm{I})$ upon staining with vancomycin-BODIPY FL showing the biofilm dimensions. Microscopy was performed with a two-photon confocal laser scanning microscope (Zeiss LSM 7MP). Images were analyzed with ImageJ, Living Image 4.7.3 and Imaris 9.5.0 software. 

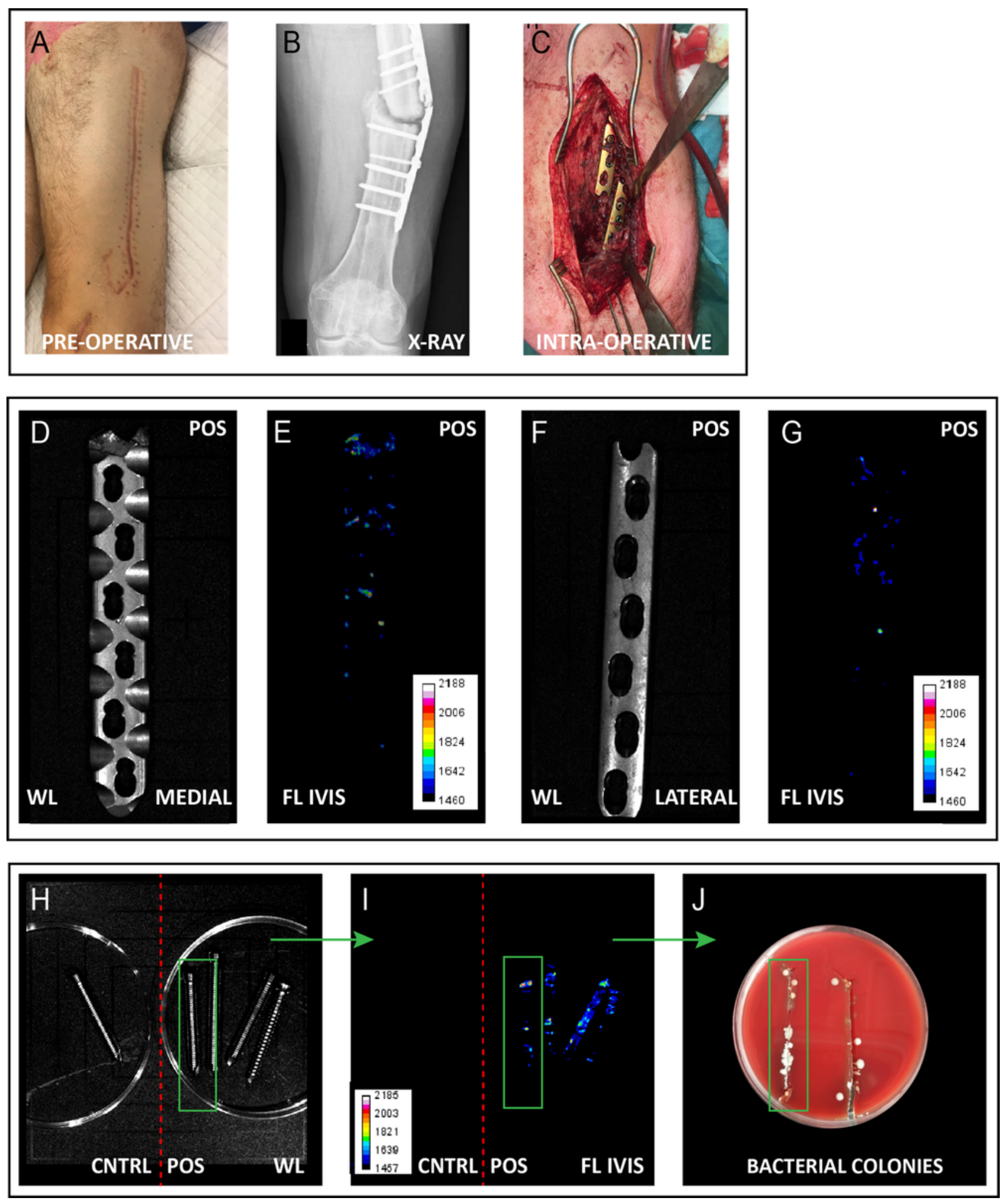

Figure 7

Bacteria-targeted fluorescence imaging and corresponding microbiological culturing of a fracture-related femur infection. Images relate to patient 5. A. Pre-operative clinical presentation with no signs of infection at the lateral thigh. B. X-ray of a non-union of a femoral fracture with a broken plate. C. Intra-operative image showing no signs of infection (e.g. no fluid, abscesses or diseased bone around the broken implant). D-G. Macroscopic white-light (WL) images of the back- and front sides of the extracted plate 
(D,F) with corresponding fluorescence images (E,G. IVIS Lumina II, Excitation 710 nm, Emission filter ICG, Exposure time $10 \mathrm{~s}) . \mathrm{H}, \mathrm{I}$. WL image $(\mathrm{H})$ and corresponding fluorescence image (I) of suspected infected (POS) and new negative control (CNTRL) screws (IVIS Lumina II, Excitation 710 nm, Emission filter ICG, Exposure time $10 \mathrm{~s}$ ). I,J. Correlation between fluorescence images (I) with bacterial colonies on a blood agar plate (J). Images were analyzed with Image J and Living Image 4.7.3 software.

A.

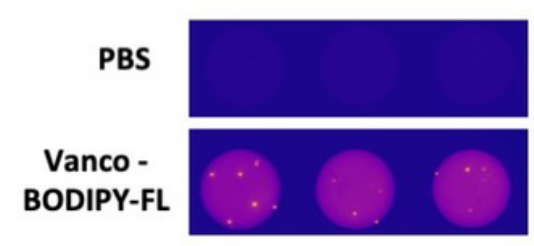

vanA-1

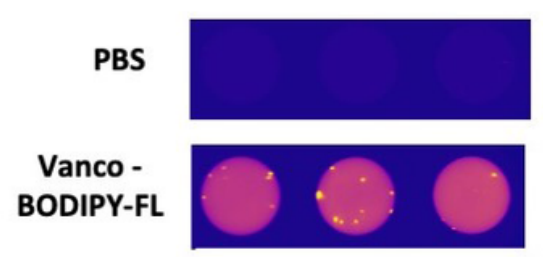

EF

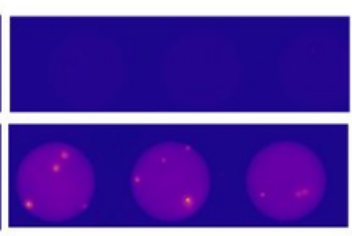

vanA-2

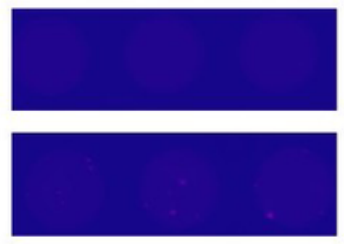

EC

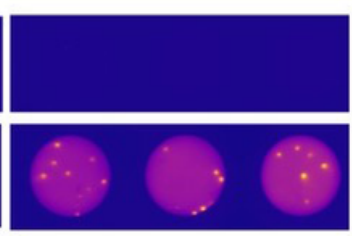

vanB-3

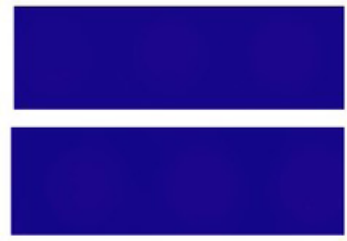

KO

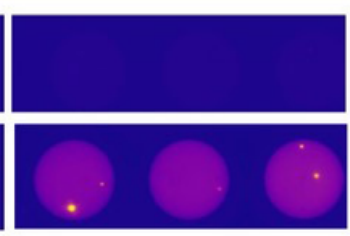

vanB-4

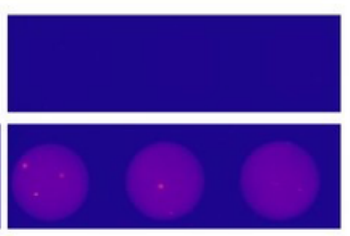

van $B-5$

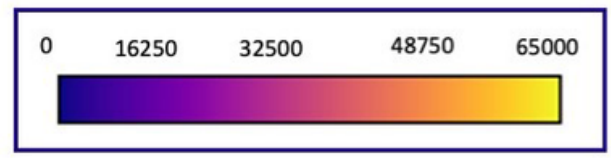

B.

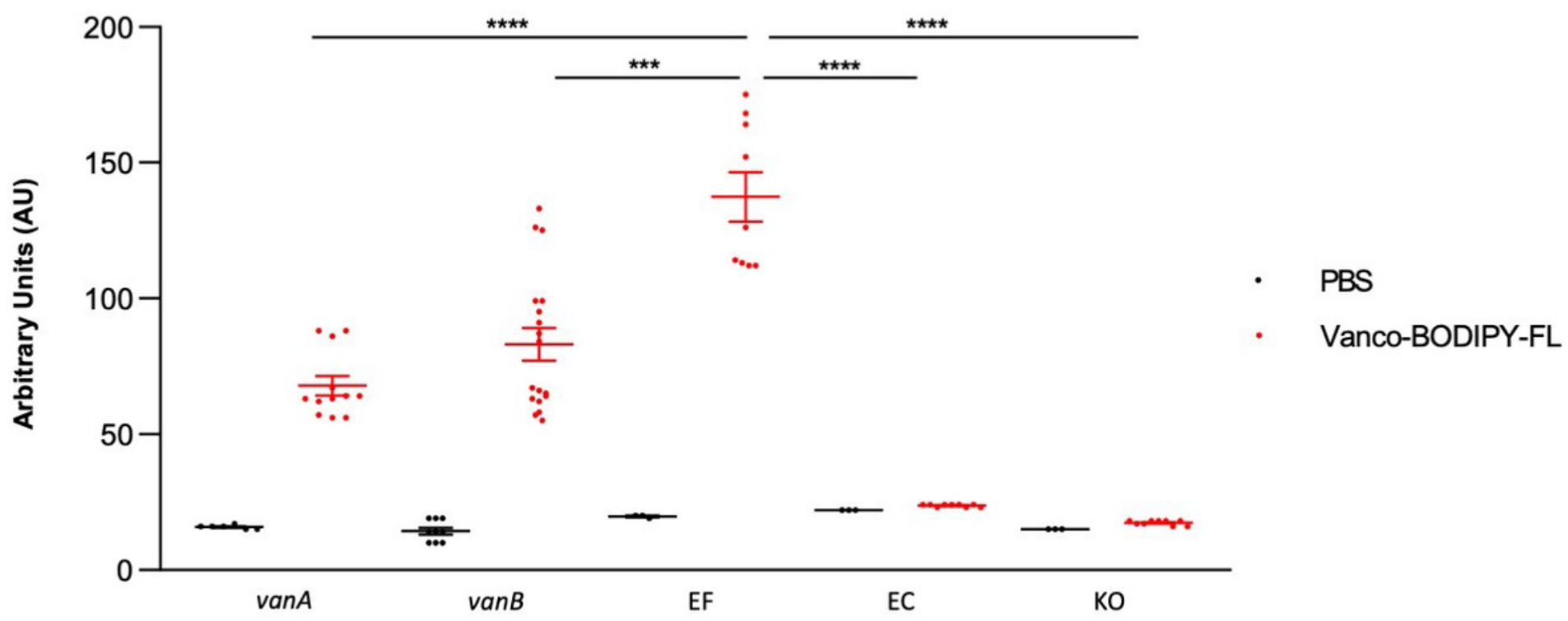

Figure 8

Binding of vancomycin-BODIPY FL by vancomycin resistant Gram-positive bacteria. A. Macroscopic imaging of vanA- or vanB-positive vancomycin resistant clinical E. faecium isolates (Gram-positive), a vancomycin sensitive clinical E. faecalis isolate (EF, Gram-positive), E. coli (EC, Gram-negative) and Klebsiella oxytoca (KO, Gram-negative) upon incubation with vancomycin-BODIPY FL and subsequent washing with PBS. Of note, the vanA-1 and vanB-5 strains still test sensitive for vancomycin with MIC 
values $\leq 0.5 \mathrm{mg} . \mathrm{L}-1$, whereas the vanA-2, vanB-3 and vanB-4 strains tests resistant for vancomycin with MIC values of $8, \geq 32$ and 8 mg.L-1, respectively, as analyzed by Vitek2 (bioMérieux). For control, PBSwashed bacteria were imaged that had not been incubated with vancomycin-BODIPY FL. Images were recorded with an Amersham Typhoon Biomolecular Imager (Filter Cy2 525BP20). B. Quantification of the vancomycin-BODIPY FL-specific fluorescence of the bacteria imaged in A in arbitrary units. The red data points mark the measurements for bacteria labelled with vancomycin-BODIPY FL, while the black data points mark controls for autofluorescence of the bacteria that were only washed with PBS. Fluorescence was measured in a Biotek Synergy 2.0 plate reader (BioTek Instruments, Inc., USA, filters optimal for FITC, excitation was set at $480 \mathrm{~nm}$ and emission at $520 \mathrm{~nm}$, with optics position at "bottom"). Mann-Whitney U

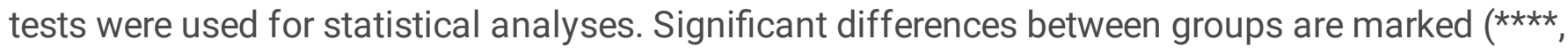
$\left.P<0.0001,{ }^{\star \star \star}, P=0.0002\right)$. Values represent the mean $\pm S E M$.

\section{Supplementary Files}

This is a list of supplementary files associated with this preprint. Click to download.

- LopezAlvarezetalMovieS1.avi 